\title{
Inhomogeneity, dynamical symmetry, and complexity in high-temperature superconductors: Reconciling a universal phase diagram with rich local disorder
}

\author{
GUIDRY Mike ${ }^{1,2}$, SUN Yang ${ }^{1,2 *} \&$ WU ChengLi ${ }^{3}$ \\ ${ }^{1}$ Department of Physics, Shanghai Jiao Tong University, Shanghai 200240, China; \\ ${ }^{2}$ Department of Physics and Astronomy, University of Tennessee, Knoxville, Tennessee 37996-1200, USA; \\ ${ }^{3}$ Physics Department, Chung Yuan Christian University, ChungLi, Taiwan 32023, China
}

Received October 9, 2010; accepted November 23, 2010

\begin{abstract}
A model for high-temperature superconductors incorporating antiferromagnetism, $d$-wave superconductivity, and no double latticesite occupancy can give energy surfaces delicately balanced between antiferromagnetic and superconducting order for specific ranges of doping and temperature. The resulting properties can reconcile a universal cuprate phase diagram with rich inhomogeneity, relate that inhomogeneity to pseudogaps, give a fundamental rationale for giant proximity effects and other emergent behavior, and provide an objective framework to separate essential from peripheral in the superconducting mechanism.
\end{abstract}

high-temperature superconductivity, pseudogap, critical dynamical symmetry, inhomogeneity, complexity, emergent behavior

Citation: Guidry M, Sun Y, Wu C L. Inhomogeneity, dynamical symmetry, and complexity in high-temperature superconductors: Reconciling a universal phase diagram with rich local disorder. Chinese Sci Bull, 2011, 56: 367-371, doi: 10.1007/s11434-010-4282-1

High-temperature superconductivity was discovered more than two decades ago [1], but its interpretation remains controversial $[2,3]$. We believe that this lack of theoretical consensus may be attributed to two fundamental, and related, issues: (1) Most models emphasize a limited aspect of the (complex) problem; as a result, there are few solvable models that capture a sufficient range of essential physics. (2) The complex behavior of these compounds obscures the superconducting mechanism because, in the absence of solvable models incorporating a sufficiently wide range of the physics, it is difficult to separate essential from secondary features using only data.

For example, high-temperature superconductors exhibit a variety of spatial inhomogeneities such as stripes or checkerboards, particularly in the hole-underdoped region and near magnetic vortex cores [4-8]. The relationship of these inhomogeneities to the unusual properties of these systems has yet to be settled. Does it lead to superconductivity (SC)?

*Corresponding author (email: sunyang@ @stu.edu.cn)
Does it oppose SC? Or is it a sideshow? Particularly vexing is that this rich variety of inhomogeneities would at first glance seem to be inconsistent with the broad evidence for a universal cuprate phase diagram, particularly for hole-doped compounds. How does one reconcile a seemingly universal phase diagram with a bewildering array of inhomogeneities for individual compounds?

Dopant atoms play a dual role in high-temperature superconductors; they support SC globally by enhancing charge carrier densities, but may suppress SC locally through atomicscale disorder. McElroy et al. [9] found strong disorder in atomically-resolved scanning tunneling microscope images of the SC gap for Bi-2212. They concluded that this disorder derives primarily from dopant impurities and that charge variations between nano-regions are small, implying that any inhomogeneity is tied to impurities and need not couple strongly to charge.

We show here that such inhomogeneities are a consequence of perturbations of the antiferromagnetic (AF) and SC correlations that are largely independent of specifics and not 
necessarily coupled to charge variations. Further, we show that these properties are consistent with a universal cuprate phase diagram, are tied intimately to the nature of pseudogap states, and imply a connection among pseudogaps, inhomogeneities, and emergent behaviors. Thus we provide a testable hypothesis for separating primary from derivative features in the rich high-temperature superconductor data set.

The SU(4) model [10-18] is a fermion many-body theory that incorporates $\mathrm{AF}$ and SC order on an equal footing, conserves spin and charge, and implies no double occupancy on the lattice (Mott insulator ground state at half filling). The effective SU(4) Hamiltonian can be expressed as

$$
H=H_{0}-\tilde{G}_{0}\left[(1-\sigma) D^{\dagger} D+\sigma \vec{Q} \cdot \vec{Q}\right]+g^{\prime} \vec{S} \cdot \vec{S},
$$

where $H_{0}, \tilde{G}_{0}$ and $g^{\prime}$ are parameters, $D^{\dagger}$ creates $d$-wave singlet pairs, $\vec{Q}$ is the staggered magnetization, $\vec{S}$ is spin, $\tilde{G}_{0}=\chi(x)+G_{0}(x)$, and

$$
\sigma=\sigma(x)=\frac{\chi(x)}{\chi(x)+G_{0}(x)},
$$

where $G_{0}(x)$ and $\chi(x)$ are effective SC and AF coupling strengths $[14,15]$. Doping is characterized by a parameter $x=$ $1-n / \Omega$ for an $n$-electron system, with $\Omega$ the maximum number of doped holes (or doped electrons for electron-doped compounds) that can form coherent pairs, taking the normal state at half filling as the vacuum. Generally, $P \simeq 0.25 x$, where $P$ is the standard hole-doping parameter, normalized to the number of copper sites [13].

Three dynamical symmetry limits have exact solutions [10-12]. The $\mathrm{SO}(4)$ limit $(\sigma=1)$ is a collective AF state, the $\mathrm{SU}(2)$ limit $(\sigma=0)$ is a collective $\mathrm{SC}$ state, and the $\mathrm{SO}(5)$ limit $(\sigma=1 / 2)$ is a critical dynamical symmetry interpolating between the SC and AF limits [10-12]. For other values of $\sigma$ an approximate solution can be obtained using the generalized coherent-state method, which is a well-developed theoretical approach relating a many-body algebraic theory with unbroken symmetry to an approximation of that theory that exhibits spontaneously broken symmetry. It has also been shown to be equivalent to the most general Hatree-FockBogoliubov variational method under symmetry constraints [10-12]. The result of such a coherent-state analysis can be presented through energy surfaces in which order parameters appear as independent variational variables. We characterize AF in these solutions by the staggered magnetization $Q \equiv\left\langle Q_{z}\right\rangle$, or by the parameter $\beta$ defined through

$$
Q=2 \Omega \beta\left(n /(2 \Omega)-\beta^{2}\right)^{1 / 2},
$$

and characterize singlet $d$-wave pairing through the gap parameter $\Delta=\left\langle D^{\dagger} D\right\rangle^{1 / 2}$.

The total energy surfaces are obtained from the expectation value of eq. (1) in the coherent state approximation. To relate these results to the experimental phase diagram we use the variation of $\sigma$ with doping, in which the doping-dependent coupling strengths $G_{0}(x)$ and $\chi(x)$ were determined by fitting to cuprate data. Figure 1 shows these strengths used in [14] that described the cuprate data. With these strengths, we calculate energy surfaces as functions of the $\mathrm{AF}$ order parameter $\beta$, the $\mathrm{SC}$ order parameter $\Delta$, and doping $x$, and show them in Figure 2. These energy surfaces exhibit two fundamental instabilities that may play a large role in the properties of cuprate superconductors. The first is an instability against condensing pairs when the system is infinitesimally doped away from half-filling. This instability is similar to the Cooper instability for normal superconductors, but generalized to doped Mott insulators, and accounts for the remarkably rapid development of supercon-

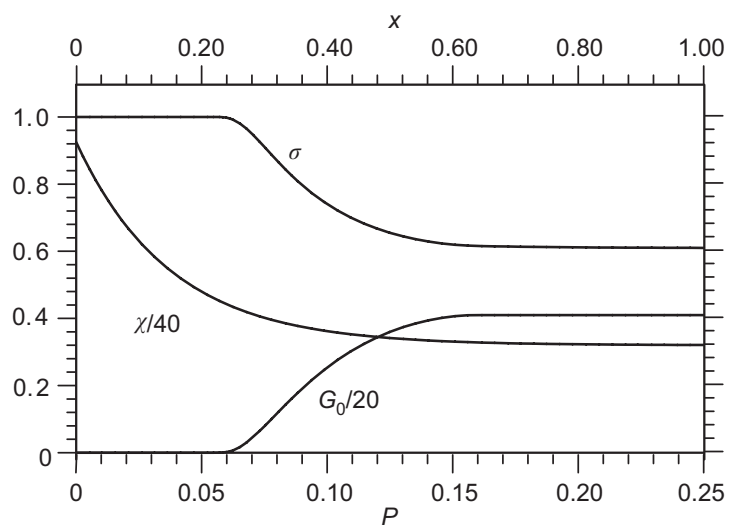

Figure 1 Variation of $\sigma=\chi /\left(\chi+G_{0}\right)$ with hole doping. Also shown are the effective coupling strengths $G_{0}(x)$ and $\chi(x)$ used in Figure 1 of [14].
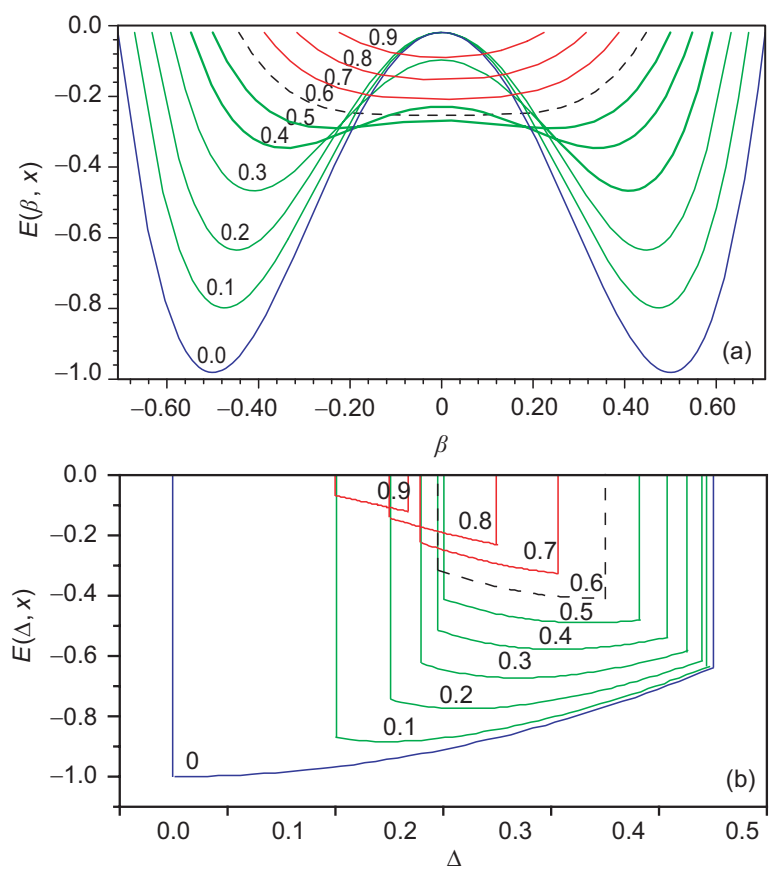

Figure 2 Total energy vs. (a) $\mathrm{AF}$ order $\beta$ and (b) SC order $\Delta$; curves labeled by hole-doping $x \simeq 4 P$, where $P$ is the number of holes per copper site. Energy is in units of $\chi \Omega^{2} / 4$, with $\chi$ the AF coupling strength. The black dashed line corresponds to critical doping $x=x_{q}$ (see $[14,15]$ ); color coding indicates energy surfaces favoring SC (red, $P=0.7-0.9$ ), AF (blue, $P=0$ ), and $\mathrm{AF}+\mathrm{SC}$ (green, $P=0.1-0.5$ ). The doping-dependent ranges of the energy contours in the order parameters reflect the finite valence space (single band) of the model. 
ductivity with hole doping in cuprates [16]. In this paper we concentrate on the properties and implications of a second fundamental instability that occurs in the underdoped region.

The energy surfaces at constant doping fall into three general classes: $\mathrm{AF}+\mathrm{SC}$ (e.g. $x=0.1$ ), SC (e.g. $x=0.9$ ), and critical (e.g. $x=0.6$, which marks a quantum phase transition). Curves in the $\mathrm{AF}+\mathrm{SC}$ class have minima at finite and large $\beta_{0}$, and small but finite $\Delta_{0}$, where the subscript zero denotes the value of the order parameter at the minimum of the energy surface. Curves in the class SC are characterized by $\beta_{0}=0$ and finite $\Delta_{0}$. Of most interest here are surfaces that are near critical in Figure 2; these correspond to broken SU(4) $\supset \mathrm{SO}(5)$ dynamical symmetry [10-12] and are flat over large regions of parameter space. This implies that there are many states lying near the ground state with very different values of $\beta$ and $\Delta$. Thus the surface is critically balanced between AF and SC order, and small perturbations can drive it from one to the other. This defines a critical dynamical symmetry of the SU(4) algebra [10-12]; we shall use the term dynamical criticality to refer to manifestation of this property in physical systems.

The extreme sensitivity of critical surfaces to perturbations is illustrated in Figure 3. Each set of curves is associated with a fixed value of doping $x=0.6$ (equivalently, $P=0.15$ ), with the solid line corresponding to $\sigma=0.6$, the dashed line to a $10 \%$ increase in $\sigma$ (AF perturbation), and the dotted line to a $10 \%$ reduction in $\sigma$ (SC perturbation). The effect on the energy surface versus $\Delta$ (not shown) is significant but less dramatic: $\Delta_{0}$ is shifted, but remains finite in all three cases. We see that this small fluctuation in $\sigma$ can alter the energy surface between $\mathrm{AF}+\mathrm{SC}$ (finite $\beta_{0}$ and $\left.\Delta_{0}\right)$ and $\mathrm{SC}\left(\beta_{0}=0\right.$ and finite $\Delta_{0}$ ). This sensitivity is specific to the critical (broken $\mathrm{SU}(4) \supset \mathrm{SO}(5)$ ) dynamical symmetry. The AF region near $x=0$ and the $d$-wave superconducting region at larger hole doping (see Figure 2) are inherently stable against such perturbations.

The AF instability displayed graphically in Figure 3 may also be understood analytically. From the $T=0$ solution for $Q$ given by eqs. (24a) and (14) of [14], we find

$$
\left.\frac{\partial Q}{\partial x}\right|_{x=x_{q}}=-\left.\frac{1}{4} \frac{x_{q}+x_{q}^{-1}-2 x}{\left[\left(x_{q}-x\right)\left(x_{q}^{-1}-x\right)\right]^{1 / 2}}\right|_{x=x_{q}}=-\infty,
$$

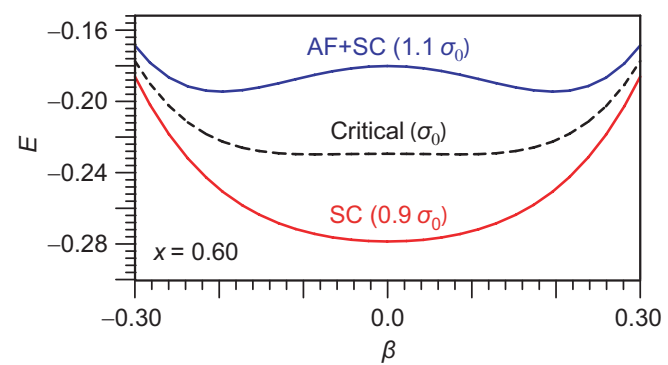

Figure 3 Energy surfaces as a function of the AF order parameter $\beta$ for fixed doping $P=x / 4=0.15$. The dashed line corresponds to $\sigma=\sigma_{0}=0.6$, the upper curve to a $10 \%$ increase in $\sigma$, and the lower curve to a $10 \%$ reduction in $\sigma$. and a small change in doping will result in a large change in antiferromagnetic correlations near $x=x_{q}$. This is a consequence of SU(4) symmetry, which requires that $Q$ vanish for $x \geqslant x_{q}$ and be finite for $0<x<x_{q}$.

It is not hard to conjecture mechanisms altering the ratio of AF to SC coupling locally. For example, [9] found that nanoscale disorder is tied to the influence of dopant impurities. Nunner et al. [19] (see also [20]) compared these results with Boboliubov-de Gennes calculations and proposed that out-of-plane dopant atoms can modulate pairing on a scale comparable to the lattice spacing, through lattice-distortion modification of electron-phonon coupling or superexchange.

To summarize, states associated with critical dynamical symmetry have variational energy surfaces that are flat over large regions of parameter space and their intrinsic collective properties may be changed qualitatively by a small perturbing background that alters the AF-SC competition. Figure 2 suggests that underdoped cuprates have near-critical energy surfaces. Thus, such states are central to the discussion of inhomogeneity and to the general issue of understanding pseudogap states in underdoped cuprates.

Figure 4 is constructed from the expectation value of (1) in the coherent state approximation, assuming a 1-D spatial perturbation, $\sin (2 \pi L)$, with $\sigma=0.6$. It illustrates schematically how a small (10\%) periodic fluctuation in the AF and SC coupling for a critically symmetric underdoped compound can lead to inhomogeneity. In this example, 1-dimensional spatial variations of the coupling ratio $\sigma$ give fluctuations in the order parameters leading to stripes in which $\mathrm{AF}+\mathrm{SC}(\sigma>0.6)$ and SC $(\sigma<0.6)$ are alternately favored. Also shown are the responses of $\mathrm{AF}$ fluctuations $\mathrm{d} \beta / \mathrm{d} L$ to this variation in $\sigma$ (We do not intend this as a realistic model of a stripe phase, but as a template indicating how such a model could be built). One plausible physical reason for a spatial $\sigma$ variation to arise may be dopant impurities, suggested in [9], and in particular magnetic dopant impurities, which will certainly affect locally the AF interaction.

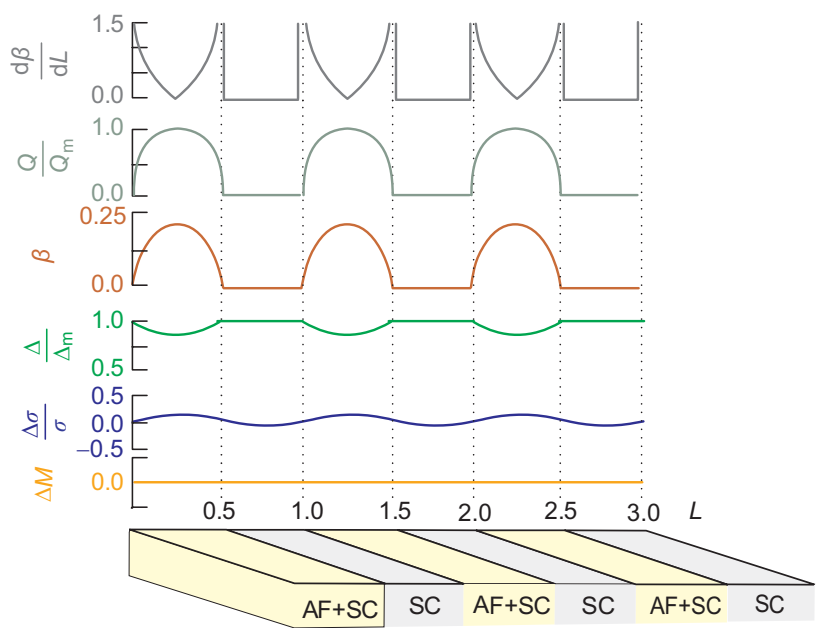

Figure 4 A small, spatially-periodic variation in the AF and SC coupling can produce inhomogeneity without significant charge variation. The charge is $M$; a subscript $m$ denotes maximum values. 
Figure 4 indicates that a small spatial variations in the coupling ratio $\sigma$ can produce regions having large $\mathrm{AF}$ and weaker SC order, interspersed with regions having significant SC correlations but no AF order. Although not plotted, the AF+SC regions also exhibit small triplet pair densities, which vanish in the SC regions. The primary fluctuation between stripes is in the AF order, which can jump between zero and the maximum allowed by the SU(4) model between adjacent stripes; the pairing-order changes are much smaller and pairing is finite in both the $\mathrm{SC}$ and $\mathrm{SC}+\mathrm{AF}$ regions. The variation in $\mathrm{d} \beta / \mathrm{d} L$ indicates that appreciable softness in AF and SC may occur on the boundaries between regions.

The expectation value of the charge $M$ is not a function of the coupling ratio $\sigma$ in the $\mathrm{SU}(4)$ coherent state, so the critical energy surface fluctuations responsible for alternating $\mathrm{AF}+\mathrm{SC}$ and SC stripes in Figure 4 cause no charge variation $(\Delta M=0)$. Data indicate that the relative charge variation for Bi-2212 surface nanoscale patches is less than $10 \%$, implying heterogeneity not strongly coupled to charge [9]. This view is supported by analyses of heat capacity and NMR data on Bi-2212 and YBCO that find a universal phase behavior for cuprates, with little static charge modulation [21, 22].

Of course, the variation in $\sigma$ could itself be due to a charge modulation. From the preceding discussion, we may expect that if a charge modulation occurs in either the AF region near half filling or the SC region at larger doping, its effect will be small because the energy surfaces are not critical there. However, if a charge modulation occurs in the underdoped region where the energy surfaces are near critical its effect could be amplified by dynamical criticality even if $\sigma$ is not altered significantly, because this is equivalent to a doping modulation. Thus, we suggest a mechanism for producing strong inhomogeneity without necessarily invoking charge fluctuations as the cause, but that can for some underdoped compounds be produced by a charge modulation. Such a mechanism could reconcile strong inhomogeneity with a universal phase diagram, and resolve competing experimental claims regarding the role of charge variation in producing inhomogeneity.

As an aside, inhomogeneity without charge modulation has an analog in astronomy. We might assume, erroneously, that density in the bright arms of a spiral galaxy (a form of "stripe order") is much larger than that between the arms. Actually, spiral arms are prominent because there mass (gravitational charge) is more visible: galactic dynamics generates many hot, luminous stars in the spiral arms. In analogy, the structure in Figure 4 is not due to charge variation but rather to modulation of the charge "visibility" by (quantum) dynamics.

The minimal patch size that can support SU(4) coherent states is crucial to the present argument. Experience (e.g. [23]) suggests that dynamical symmetry can be realized in fermion valence spaces having as few as several particles. This would be consistent with inhomogeneities on scales comparable to atomic dimensions, as required by data [9].

Inhomogeneity caused by electronic self-organization is often contrasted with that caused by dopant impurities. Our discussion implicates both as sources of nanoscale structure. The proximate cause may be impurities that perturb the SU(4) energy surface but the criticality of that surface, which greatly amplifies the influence of impurities, results from the selforganizing, doped Mott insulator encoded in the SU(4) algebra. Note an analysis [20] suggesting, from a different perspective, that intrinsic amplification of impurity effects is required to explain nanoscale structure in Bi-2212.

Critical dynamics may also produce inhomogeneities near magnetic vortices and magnetic impurities. A magnetic field should suppress $\mathrm{SC}$ relative to $\mathrm{AF}$, so the distance from a vortex $d$ may be expected to alter the average value of $\sigma$, just as changing the doping $P$ would. Therefore, we may expect a region near magnetic vortices or impurities where the symmetry is critical and exhibits sensitivity to perturbations similar to that exhibited in Figure 3, with $d$ modulating $\sigma$ rather than $P$.

Giant proximity effects are observed in cuprates where non-SC copper oxide material sandwiched between superconducting material can carry a supercurrent, even for a thickness much larger than the coherence length [24]. Phenomenology indicates that pre-existing nanoscale SC patches can precipitate such effects [25]. We find similar possibilities on fundamental grounds, but also suggest that the inhomogeneity need not pre-exist. Dynamical criticality renders even a homogeneous pseudogap phase unstable against large fluctuations in the AF and SC order. Thus, proximity of superconducting material to pseudogap material coupled with perturbations from background impurity fields can trigger dynamical nucleation of nanoscale structure and giant proximity effects, even if no static inhomogeneity exists beforehand.

In experiments with one unit cell thickness $\mathrm{La}_{2} \mathrm{CuO}_{4}$ AF barrier layers between superconducting $\mathrm{La}_{1.85} \mathrm{Sr}_{0.15} \mathrm{CuO}_{4}$ samples, Bozovic et al. [26] found that the two phases did not mix, with the barrier layer completely blocking a supercurrent. Interpretation of these results ruled out many models of high-temperature superconductors, in particular models in which SC and AF phases are nearly degenerate like the $\mathrm{SO}(5)$ model [27]. The absence of a proximity effect between the $\mathrm{AF}$ and SC phases (but presence of a strong proximity effect between pseudogap and SC material) is plausibly consistent with the SU(4) model: the AF phase is not rotated directly into the SC phase but rather evolves with increased doping into a mixed $\mathrm{SC}$ and $\mathrm{AF}$ phase, which then is transformed into a pure SC state at a critical doping point near optimal doping, where the AF correlations vanish identically [14, 15].

The spontaneous appearance of properties that do not preexist in a system's elementary components is termed emergence; systems displaying emergent behavior are said to exhibit complexity (see Dagotto [28] for a review). Complexity can occur when the choice between potential ground states is sensitive to even weak external perturbations. The amplification effect implied by SU(4) dynamical criticality can facilitate emergent behavior and complexity. The giant proximity 
effect and the perturbatively-induced structure of Figure 4 are examples. More generally, critical dynamical symmetry may represent a fundamental organizing principle for complexity in strongly-correlated fermionic matter. For example, critical dynamical symmetries are known in nuclear physics [29, 30].

The SU(4) coherent state method that we employ here to obtain the energy surfaces admits quasiparticle solutions that generalize the BCS equations. These give a rich, highly universal phase diagram in agreement with much of the available data $[14,15]$, and a quantitative description of Fermi arcs [17]. Therefore, the propensity of the pseudogap state to generate a broad variety of induced inhomogeneities, and a quantitative model of the cuprate phase diagram (including pseudogaps) that exhibits highly universal character, both follow directly from a model that implements $\mathrm{AF}$ and $d$-wave SC competition in a doped Mott insulator. This natural coexistence of a universal phase diagram with a rich susceptibility to disorder could reconcile many seemingly disparate observations in the cuprate superconductors. For example, because the SU(4) pseudogap state has both pairing fluctuations and critical dynamical symmetry, it could support Nernst vortex states as perturbations on a homogeneous phase having incipient nanoscale heterogeneity.

In summary, competing antiferromagnetism and $d$-wave pairing, constrained by no double site occupancy, can lead to energy surfaces in hole-underdoped cuprates that are critically balanced between antiferromagnetism and superconductivity. These surfaces can be flipped between dominance of one or the other by small changes in the ratio of the antiferromagnetic to pairing strength. Therefore weak perturbations in the underdoped region, and near vortex cores or magnetic impurities, can produce amplified inhomogeneity having the spatial dependence of the perturbation but the intrinsic character of an SU(4) symmetry (The symmetry defines the possible states; the perturbation selects from among them). Our results show that such effects could, but need not, imply spatial modulation of charge. More generally, we have suggested that critical dynamical symmetry may be a fundamental principle of emergent behavior in correlated fermion systems.

The generality of our solution implies that any realistic theory describing superconductivity competing with antiferromagnetism should contain features similar to those discussed here. In particular, similar phenomena may be possible in the new iron-based high-temperature superconductors [31], because we have proposed that they may also exhibit SU(4) dynamical symmetry [32]. Thus, the existence of complex inhomogeneities for compounds having (paradoxically) universal phase diagrams suggests that (1) properties of superconductors in the pseudogap state, and near magnetic vortices and impurities, are largely determined by critical dynamical symmetry, and (2) inhomogeneity provides a strong diagnostic tool in detailing the mechanism of hightemperature superconductivity but it is only consequential, not causative.

We thank Takeshi Egami, Elbio Dagotto, Adriana Moreo, Pengcheng Dai, and Boris Fine for discussions. M.G. and C.-L.W. are grateful for support from the SJTU-INS research project for visiting professors of Shanghai Jiao Tong University.

1 Bednorz J G, Müller K A. Z Phys B, 1986, 64: 189-193

2 Bonn D A. Nature Phys, 2006, 2: 159-168 and references therein

3 Norman M R, Pépin C. Rep Prog Phys, 2003, 66: 1547-1610 and references therein

4 Hayden S M, Mook H A, Dai P, et al. Nature, 2004, 429: 531-534

5 Tranquada J M, Woo H, Perring T G, et al. Nature, 2004, 429: 534-538

6 Hoffman J E, Hudson E W, Lang K M, et al. Science, 2002, 295: 466469

7 Vershinin M, Misra S, Ono S, et al. Science, 2004, 303: 1995-1998

8 Hanaguri T, Lupien C, Kohsaka Y, et al. Nature, 2004, 430: 1001-1005

9 McElroy K, Lee J, Slezak J A, et al. Science, 2005, 309: 1048-1052

10 Guidry M W. Rev Mex Fisica, 1999, 45(Suppl 2): 132

11 Guidry M W, Wu L A, Sun Y, et al. Phys Rev B, 2001, 63: 134516

2 Wu L A, Guidry M W, Sun Y, et al. Phys Rev B, 2003, 67: 014515

3 Guidry M W, Sun Y, Wu C L. Phys Rev B, 2004, 70: 184501

4 Sun Y, Guidry M W, Wu C L. Phys Rev B, 2006, 73: 134519

Sun Y, Guidry M W, Wu C L. Phys Rev B, 2007, 75: 134511

6 Guidry M W, Sun Y, Wu C L. Front Phys China, 2010, 5: 171-175

7 Guidry M W, Sun Y, Wu C L. New J Phys, 2009, 11: 123023

8 Sun Y, Guidry M W, Wu C L. Phys Rev B, 2008, 78: 174524

19 Nunner T S, Anderson B M, Melikyan A, et al. Phys Rev Lett, 2005 95: 177003

20 Fang A C, Capriotti L, Scalapino D J, et al. Phys Rev Lett, 2006, 96 : 017007

21 Loram J W, Tallon J L, Liang W Y. Phys Rev B, 2004, 69: 060502(R)

22 Bobroff J, Alloul H, Ouazi S, et al. Phys Rev Lett, 2002, 89: 157002

23 Wu C L, Feng D H, Guidry M W. Adv Nucl Phys, 1994, 21: 227

24 Bozovic I, Logvenov G, Verhoeven M A J, et al. Phys Rev Lett, 2004, 93: 157002

25 Alvarez G, Mayr M, Moreo A, et al. Phys Rev B, 2005, 71: 014514

26 Bozovic I, Logvenov G, Verhoeven M A J, et al. Nature, 2003, 422: 873-875

27 Demler E, Berlinsky A J, Kallin C, et al. Phys Rev Lett, 1998, 80: 2917

28 Dagotto E. Science, 2005, 309: 257-262

29 Zhang W M, Feng D H, Ginocchio J N. Phys Rev Lett, 1987, 59: 2032

30 Zhang W M, Feng D H, Ginocchio J N. Phys Rev C, 1988, 37: 1281

31 Sun Y, Guidry M W, Wu C L. Chinese Sci Bull, 2008, 53: 1617-1618

32 Guidry M W, Sun Y, Wu C L. Front Phys China, 2009, 4: 433-446 